\title{
Automatic Aortic Root Segmentation with Shape Constraints and Mesh Regularisation
}

Robert leuan Palmer

Xianghua Xie

Gary Tam
Department of Computer Science

Swansea University

Swansea, UK

csvision.swan.ac.uk
A non-invasive procedure called Transcatheter Aortic Valve Implantation (TAVI) has emerged as an alternative procedure for patients suffering with aortic stenosis, but cannot undergo standard open-heart surgery. A full segmentation of the aortic root is important to the success of the procedure, and is essential for patient selection, procedural planning, and post-evaluation [1].

We propose a fully-automatic, deformable model-based method to segment the aortic root in 3D cardiac CT images. This consists of aligning an initial mesh with an initial aortic root pose estimation, before deforming the mesh towards the object boundary in the image. The estimation of the aortic root pose can be considered as an object detection problem, and a marginal space learning (MSL) method is adopted from [3] for this purpose. Once the initial mesh is aligned with the estimated pose, we implement a two-stage mesh deformation method: non-iterative boundary segmentation followed by iterative boundary refinement with mesh smoothing. Figure 1 outlines the steps taken at the testing stage for our automatic segmentation method.

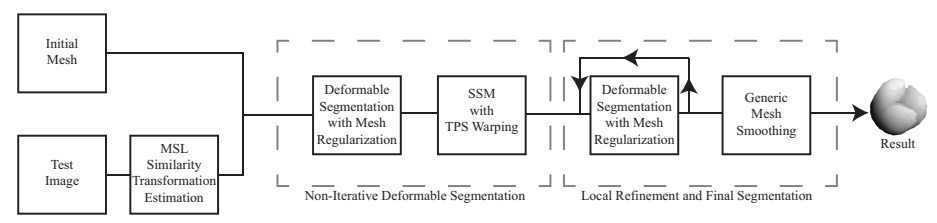

Figure 1: Overview of the proposed automatic aortic root segmentation at the testing stage.

The initial non-iterative process consists of deformable segmentation with mesh regularisation followed by a statistical-shape-model (SSM) based regularisation with thin-plate-spline (TPS) warping. The deformable segmentation consists of a learning-based boundary detector, where each vertex of the mesh template is adjusted to fit the object boundary along the orthogonal direction. After boundary detection, we have a set of original mesh vertices $V$, and a new set of vertex positions $V^{\prime}$ which may result in tangled and overlapping mesh faces. To address this problem, we propose a $B$-spline based mesh regularisation method which estimates a non-rigid transformation between $V$ and $V^{\prime}$ before performing a freeform-deformation (FFD) on $V$ to fit $V^{\prime}$. This is estimated by warping an underlying voxel lattice controlled by a set of control points $\phi_{i, j, k}^{h}$, which act as parameters of a B-spline.

$$
T(x, y, z)=\sum_{l=0}^{3} \sum_{m=0}^{3} \sum_{n=0}^{3} B_{l}(u) B_{m}(v) B_{n}(w) \phi_{i+l, j+m, k+n}
$$

where $B_{l}$ represents the $l$ th basis function of the B-spline, $i, j$ and $k$ are the corresponding lattice positions and $u, v$ and $w$ are the fractional positions along the lattice [2]. In addition, this non-rigid transformation is estimated in a multi-resolution procedure which is expressed as a summation of FFDs at multiple resolutions $\mathrm{H}$ :

$$
T^{H}(x, y, z)=\sum_{h=1}^{H} T^{h}(x, y, z)
$$

This is followed by an SSM based regularisation with TPS warping, which makes the process capable of large vertex deformations without introducing mesh irregularities.

Finally, a boundary refinement iterating a local deformable segmentation with mesh regularisation process is applied. This process again combines the learning-based boundary detector and B-spline based mesh regularisation. Finally, generic mesh smoothing is used to remove any faceted mesh faces.
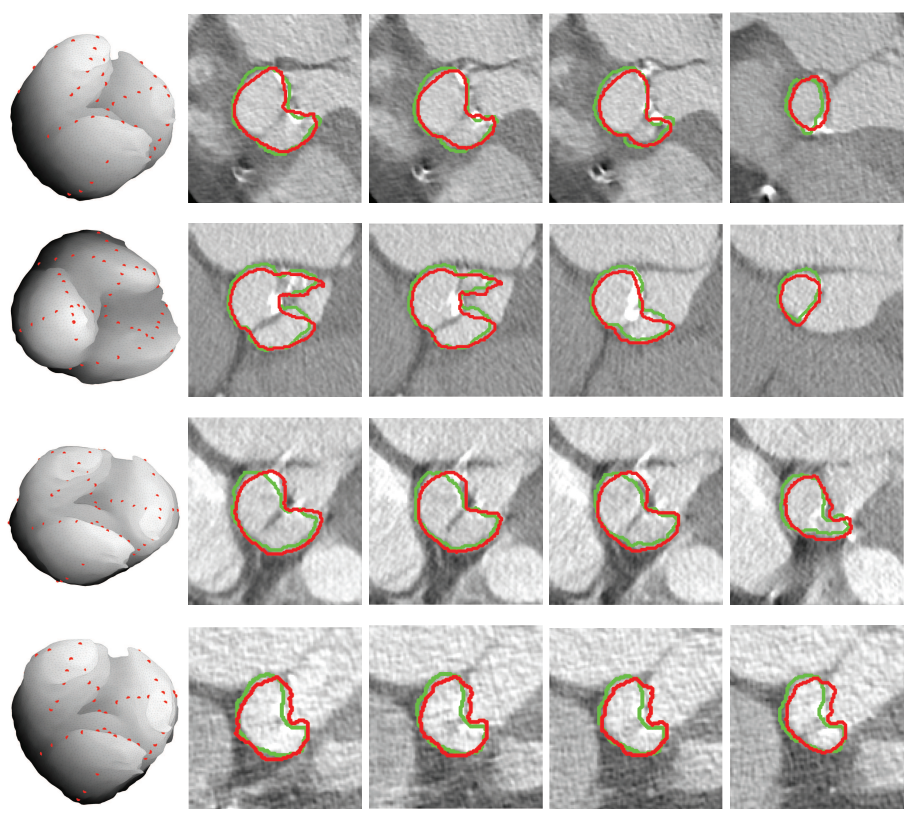

Figure 2: Results of the proposed method. Each row is a result of a different test image. The first column shows the resulting mesh. The following columns show a selection of image slices with the segmentation results. Green contours are the ground truth, while red contours are the segmentation results.
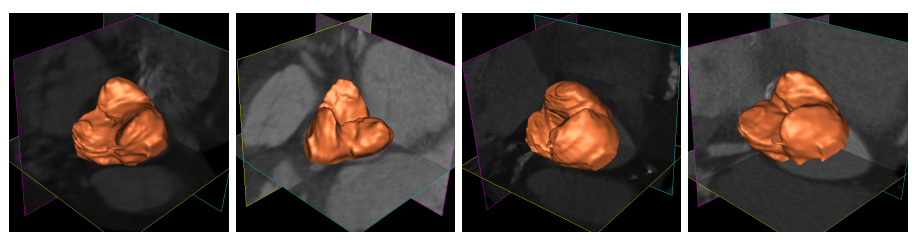

Figure 3: Further example segmentation results with our proposed method.

Quantitative comparisons were carried out against a state-of-the-art deformable model-based approach [3] and an active-shape-model based segmentation. Example segmentation results of the proposed method are shown in Figures 2 and 3. The proposed method achieves and average mesh error of $1.39 \pm 0.29 \mathrm{~mm}$, and Hausdorff distance of $6.75 \pm 2.05 \mathrm{~mm}$. These errors are lower than the two comparison approaches, and also results in much more regularised mesh surfaces with no tangled mesh faces. The proposed method is not only more efficient but also more accurate in segmenting complex anatomical structures with ambiguous image appearance.

[1] M. Gessat, T. Frauenfelder, L. Altwegg, J. Grunenfelder, and V. Falk. Transcatheter aortic valve implantation: Role of imaging. Aswan Heart Centre Science and Practice Series, 1(3), 2011.

[2] D. Rueckert, L. I. Sonoda, C. Hayes, D. Mill, O. Leach, and D. J. Hawkes. Nonrigid registration using free-form deformations: Application to breast mr images. Trans. Med. Imaging, 18(8):712-721, 1999.

[3] Y. Zheng, M. John, R. Liao, A. Nottling, J. Boese, J. Kempfert, T. Walther, G. Brockmann, and D. Comaniciu. Automatic aorta segmentation and valve landmark detection in c-arm ct for transcatheter aortic valve implantation. Trans. Med. Imaging, 31:2307-2321, 2012. 